\title{
Prävalenz chronischer Trommelfellperforationen in der erwachsenen Bevölkerung
}

\section{HINTERGRUND}

Trommelfellperforationen verheilen in der Regel spontan. Jedoch können verschiedene Faktoren zu Störungen der Wundheilungsprozesse und damit zu einer chronischen Perforation führen. Folgen einer nicht abheilenden Trommelfellperforation können Schwerhörigkeit und rezidivierende Mittelohrentzündungen sein. Deshalb wird häufig der mikrochirurgische Trommelfellverschluss angestrebt. Jedoch sind Rezidivperforationen möglich. Des Weiteren gibt es Patienten, die eine Operation ablehnen oder bei denen z. B. wegen eines eingeschränkten Allgemeinzustandes eine Operation nicht durchgeführt wird. Aus diesen Gründen wird gegenwärtig nach Wegen gesucht, durch Medikamente die Induktion eines Perforationsverschlusses zu erreichen bzw. die Wundheilung nach mikrochirurgischer Trommelfellrekonstruktion zu unterstützen (z.B. Einsatz von Wachstumsfaktoren) [1]. Um den quantitativen Bedarf für solche neuartigen Therapiemodalitäten abschätzen zu können, ist die Bestimmung der Prävalenz chronischer Trommelfellperforationen notwendig.

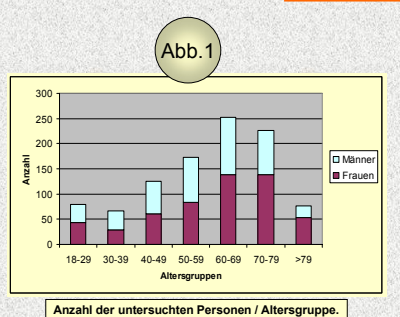

Anzahl der untersuchten Personen / Altersgruppe.

\section{MATERIAL UNA METHODEN}

Die Studie wurde durch die Ethikkommission der Ärztekammer Mecklenburg-Vorpommern der Ernst-Moritz-Arndt-Universität Greifswald genehmigt. Von März 2004 bis Februar 2006 wurden 1.000 freiwillige Personen (547 Frauen, 453 Männer) im Alter von 18 bis 93 Jahren untersucht (Abb. 1). Die Probanden wurden zufällig aus dem Patientengut dreier Kliniken der Ernst-MoritzArndt-Universitat und aus zwei Greifswalder Arztpraxen ausgewahilt, in welchen sie auf Grund nicht HNO-arztlicher Beschwerden in Behandlung waren. Unter Nutzung eines einheitlichen Respirationstraktes. Nasenatmungsbehinderung, Sodbrennen, Einnahme von immunsupprimierenden Medikamenten, Berufstätigkeit und Schadstoffbelastung erhoben. Es wurde eine

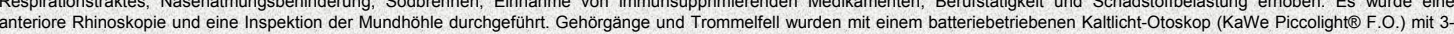
facher Vergrößerung untersucht. Für die Angabe der standardisierten Prävalenz chronischer Trommelfellperforationen erfolgte die Angleichung der Studienpopulation an die Alters- und Geschlechtsverteilung der ,Europäischen Standardbevölkerung". Des Weiteren wurden die Häufigkeiten potentieller Risikofaktoren in der Gruppe der Probanden mit Perforation denen in der Probandengruppe ohne Perforation gegenübergestellt. Die Beurteilung bezüglich eines signifikanten Unterschiedes erfolgte mit Hilfe des Fisher's exact-Test. Ein $p$-Wert $<0,05$ wurde als statistisch signifikant definiert. Alle statistischen Analysen wurden mit SPSS, Version 14.0.1 (Chicago, II, USA) durchgeführt.

\section{ERGEBNISSE}

Von den 1.000 untersuchten Personen wiesen 8 (4 Männer, 4 Frauen) eine einseitige und ein weiterer Mann eine beidseitige chronische Trommelfellperforation auf (Tab. 1). Für die 9 Probanden mit chronischer Trommelfellperforation wurde nach Altersstandardisierung eine Gesamtprävalenz von 0,45 \% (Konfidenzintervall: 0,03 - 0,87) ermittelt. Das Durchschnittsalter dieser Personen zum Zeitpunkt der Untersuchung betrug 67,2 Jahre, die mittlere Perforationsdauer 24,5 Jahre. Bei allen Probanden trat die Perforation, die sich im weiteren Verlauf nicht mehr verschließen sollte, im Rahmen einer akuten Otitis media auf. Bei 8 Trommelfellen war die Perforation eine direkte Folge des entzündlichen Prozesses, bei 2 Trommelfellen persistierte eine zur Behandlung der akuten Mittelohrentzündung durchgeführte Parazenteseöffnung (davon ein Mal mit Einsetzen eines Paukenröhrchens). Bei 5 Probanden mit Trommelfellperforation lag ein aktuelles Tonaudiogramm vor, welches für das Ohr mit Trommelfellperforation eine kombinierte Schwerhörigkeit bei 4 und eine Surditas bei einem Probanden zeigte. Bei einem 63jährigen Probanden war in der Kindheit eine Ohroperation (vermutlich eine Antrotomie) durchgeführt worden. 40 Jahre später trat auf diesem Ohr die bis zum Untersuchungszeitpunkt persistierende Perforation auf.

Die Häufigkeit der Symptome Hörminderung, Tinnitus, Otorrhoe sind in Tab.1 aufgeführt. Rezidivierende Otorrhoen traten meist im Rahmen von Infekten der oberen Atemwege auf. Bei keinem der 1.000 Probanden wurde ein Cholesteatom nachgewiesen, jedoch stellte sich bei 6 Probanden einseitig eine offene Mastoidhöhle / Radikalhöhle nach chirurgischer Sanierung eines Cholesteatoms dar.

In Tab.2 werden ausgewählte anamnestische und klinische Befunde aufgeführt und deren Häufigkeitsverteilung zwischen der Gruppe der Probanden mit chronischer Trommelfellperforation und der ohne Perforation verglichen.

\begin{tabular}{|c|c|c|c|c|c|c|}
\hline \multirow{2}{*}{ Tab.1 } & $\begin{array}{c}\text { Geschlecht } / \\
\text { Alter (J.) }\end{array}$ & $\begin{array}{c}\text { Seite der } \\
\text { Perforation }\end{array}$ & $\begin{array}{c}\text { Perforations- } \\
\text { dauer (J.) }\end{array}$ & Hörminderung & Tinnitus & Otorrhoe \\
\hline 1 & $\mathrm{~m} / 48$ & links & 5 & + & + & + \\
\hline 2 & $\mathrm{~m} / 62$ & rechts & 54 & + & - & + \\
\hline 3 & $\mathrm{~m} / 63$ & rechts & 11 & + & - & + \\
\hline 4 & $\mathrm{~m} / 65$ & beidseitig & $0.5 / 0.5$ & $+/+$ & $-/-$ & $+/+$ \\
\hline 5 & $\mathrm{w} / 66$ & rechts & 40 & + & + & - \\
\hline 6 & $\mathrm{w} / 66$ & links & 10 & - & + & + \\
\hline 7 & $\mathrm{~m} / 70$ & rechts & 60 & + & - & - \\
\hline 8 & $\mathrm{w} / 81$ & rechts & 63 & + & - & - \\
\hline 9 & $\mathrm{w} / 84$ & rechts & 1 & + & - & - \\
\hline Gesamt & $\mathbf{5} \mathrm{m} / \mathbf{4} \mathbf{w}$ & $\begin{array}{c}\mathbf{3} \text { links } \\
\mathbf{7} \text { rechts }\end{array}$ & $\mathbf{2 5 . 5}$ & $\mathbf{9 0} \%$ & $\mathbf{3 0} \%$ & $\mathbf{6 0} \%$ \\
\hline
\end{tabular}

\begin{tabular}{|c|c|c|c|c|c|}
\hline \multicolumn{1}{c|}{ Tab.2 } & $\begin{array}{c}\text { Probanden mit } \\
\text { cTFP ( } \mathbf{n}=\mathbf{9})\end{array}$ & $\begin{array}{c}\text { Probanden ohne } \\
\text { CTFP (n=991) }\end{array}$ & Odds Ratio & KI & $\mathbf{p}$ \\
\hline $\begin{array}{c}\text { AOM im } \\
\text { Erwachsenenalter }\end{array}$ & $5(55,6 \%)$ & $82(8,3 \%)$ & 13,86 & $3,65-52,61$ & $<0.001$ \\
\hline $\begin{array}{c}\text { AOM in der } \\
\text { Kindheit }\end{array}$ & $5(55,6 \%)$ & $242(24,4 \%)$ & 3,87 & $1,03-14,52$ & 0.046 \\
\hline Immunsuppression & $2(22,2 \%)$ & $32(3,2 \%)$ & 8,56 & $1,71-42,86$ & 0.035 \\
\hline $\begin{array}{c}\text { Rez. Infekte der } \\
\text { oberen Atemwege }\end{array}$ & $2(22,2 \%)$ & $79(7,9 \%)$ & 3,3 & $0,67-16,14$ & 0.16 \\
\hline Exponierter Beruf & $4(44,4 \%)$ & $274(27,6 \%)$ & 2,09 & $0,56-7,68$ & 0.27 \\
\hline Diabetes mellitus & $2(22,2 \%)$ & $184(18,6 \%)$ & 1,25 & $0,26-6,28$ & 1.966 \\
\hline
\end{tabular}

\section{DISKUSSION}

Die bisher wenigen Angaben zur Prävalenz chronischer Trommelfellperforationen bei Erwachsenen in Europa stammen zumeist aus älteren Studien oder können nur aus allgemeinen Prävalenzdaten zu chronischen Mittelohrentzündungen abgeschätzt werden. So ergab eine aktuelle Studie aus Norwegen eine Prävalenz von $1,5 \%$ für chronische Mittelohrentzündungen, ohne das dabei zwischen trockener Trommelfellperforation, chronischer Schleimhauteiterung, Cholesteatom sowie deren Folgen (z.B. Radikalhöhle, vernarbtes Trommelfell) differenziert wurde [2]. Die von uns ermittelte, sich nur auf chronische Trommelfellperforationen beziehende alterstandardisierte Prävalenz von $0,45 \%$ fällt erwartungsgemäß niedriger aus.

Eine gestörte Tubenfunktion begünstigt das Auftreten von rezidivierenden akuten Mittelohrentzündungen und scheint ein prädisponierender Faktor für eine ausbleibende Trommelfellwundheilung zu sein [3]. Dies könnte erklären, dass in der vorliegenden Studie Personen mit chronischer Trommelfellperforation signifikant häufiger von wiederkehrenden akuten Mittelohrentzündungen berichteten als Personen ohne Perforation.

Eine Tympanoplastik lehnten 8 der 9 Probanden mit chronischer Trommelfellperforation ab. Als Gründe für diese Entscheidung wurden Angst vor einer Operation; fortgeschrittenes Alter; bereits durchgeführte, aber erfolglose Tympanoplastik aufgeführt. Dies unterstreicht, dass Forschungsprojekte zur nichtchirurgischen Induktion des Trommelfellverschlusses klinisch relevant sind. Durch die in dieser Studie ermittelten Daten zur Prävalenz chronischer Trommelfellperforationen kann der quantitative Bedarf für neuartige Therapie-Modalitäten abgeschätzt werden.

\section{SCHLUSSFOLGERUNGEN}

Der hohe Anteil an Personen mit chronischer Trommelfellperforation, die nicht operiert werden möchten, verdeutlicht, dass ein großer Bedarf an neuartigen Therapieverfahren besteht.

\section{LITERATUR}

Kaftan H, Hosemann W, Junghans D, Göpferich A, Schindler E, Beule A (2005) Der Einfluß einer den Wachstumsfaktor EGF freisetzenden Folie auf die Heilung einer akuten Trommelfellperforation im Tiermodell. HNO 53: 539-544 